\title{
Remitted Major Depression is Characterized by Reduced Prefrontal Cortex Reactivity to Reward Loss
}

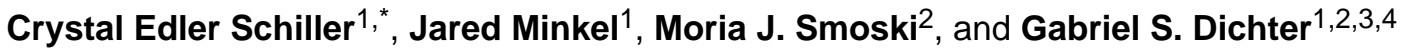 \\ ${ }^{1}$ Department of Psychiatry, University of North Carolina at Chapel Hill School of Medicine, Chapel \\ Hill, NC 27599, United States \\ 2Department of Psychiatry and Behavioral Sciences, Duke University Medical Center, Durham, \\ NC 27710, United States \\ ${ }^{3}$ Carolina Institute for Developmental Disabilities, University of North Carolina at Chapel Hill \\ School of Medicine, Chapel Hill, NC 27599, United States \\ ${ }^{4}$ Duke-UNC Brain Imaging and Analysis Center, Duke University Medical Center, Durham, NC \\ 27710, United States
}

\begin{abstract}
Background-Major depression (MDD) is characterized by anhedonia. Although a growing body of literature has linked anhedonia in MDD to reduced frontostriatal activity during reward gains, relatively few studies have examined neural responsivity to loss, and no studies to date have examined neural responses to loss in euthymic individuals with a history of MDD.
\end{abstract}

Methods-An fMRI monetary incentive delay task was administered to 19 participants with remitted MDD (rMDD) and 19 never depressed controls. Analyses examined group activation differences in brain reward circuitry during monetary loss anticipation and outcomes. Secondary analyses examined the association between self-reported rumination and brain activation in the rMDD group.

Results-Compared to controls, the rMDD group showed less superior frontal gyrus activation during loss anticipation and less inferior and superior frontal gyri activation during loss outcomes (cluster corrected $p$ 's<.05). Ruminative Responses Scale scores were negatively correlated with superior frontal gyrus activation $(r=-.68, p=.001)$ during loss outcomes in the rMDD group. Limitations: Replication with a larger sample is needed.

Conclusions-Euthymic individuals with a history of MDD showed prefrontal cortex hypoactivation during loss anticipation and outcomes, and the degree of superior frontal gyrus hypoactivation was associated with rumination. Abnormal prefrontal cortex responses to loss may

(C) 2013 Elsevier B.V. All rights reserved.

*Corresponding author at: Department of Psychiatry, University of North Carolina at Chapel Hill School of Medicine, CB\# 7175, 101 Manning Drive, Chapel Hill, NC 27599-3366, United States. Tel.: +1 919966 4810; fax: +1 9199660708.

crystal_schiller@med.unc.edu (C.E. Schiller).

All authors declare that they have no conflicts of interest.

Drs. Dichter and Smoski designed the study, wrote the protocol, and assisted with manuscript revisions. Dr. Schiller conducted the statistical analyses and wrote the first draft of the manuscript. Dr. Minkel assisted with the statistical analyses and manuscript revisions. All authors contributed to and have approved the final manuscript.

Publisher's Disclaimer: This is a PDF file of an unedited manuscript that has been accepted for publication. As a service to our customers we are providing this early version of the manuscript. The manuscript will undergo copyediting, typesetting, and review of the resulting proof before it is published in its final citable form. Please note that during the production process errors may be discovered which could affect the content, and all legal disclaimers that apply to the journal pertain. 
reflect a trait-like vulnerability to MDD, although future research is needed to evaluate the utility of this functional neural endophenotype as a prospective risk marker.

\section{Keywords}

Major depressive disorder; Remission; Reward; Rumination; Anticipation; Magnetic resonance imaging

\section{Introduction}

Major depressive disorder (MDD) is characterized both by negative mood and anhedonia, the lack of interest or pleasure in usually rewarding activities. Although a growing body of literature has linked anhedonia to reduced frontostriatal activity during reward gains in MDD (Forbes et al., 2009; Keedwell et al., 2005; Knutson et al., 2008; Mitterschiffthaler et al., 2003; Schaefer et al., 2006; Smoski et al., 2011), relatively few studies have examined neural responsivity to loss. This is a critical omission given that largely overlapping brain regions respond to reward gains and losses (Knutson and Greer, 2008) and that different forms of psychopathology are associated with unique patterns of neural deficits when processing reward gains and losses (Choi et al., 2012).

Knutson et al. (Knutson et al., 2008) investigated neural responses to reward loss with functional magnetic resonance imaging (fMRI) and found relatively reduced reactivity across multiple brain regions to loss in patients with MDD, including middle frontal gyri, parietal cortex, sublenticular extended amygdala, and putamen. Given the clinical relevance of processing losses for MDD and MDD risk (Kendler and Hettema, 2003; Nolen-Hoeksema et al., 1994), a critical question is whether abnormal neural responses to losses reflect a state-dependent abnormality consequent to active depressive symptoms or a trait marker of vulnerability to MDD. However, no study to date has examined neural correlates of loss processing in euthymic individuals with a history of MDD.

The purpose of the current study was to examine the neural correlates of loss processing in individuals with remitted major depression (rMDD). Although such a design is not sufficient to conclusively establish the neural abnormalities that preceded the onset of MDD, it allows for an examination of associations between depressogenic risk factors and patterns of brain function while mitigating the potential confounding effect of current mood state, illness severity, nonspecific effects of chronic illness and stress, and the effects of psychotropic medication (Kerestes et al., 2012). Ultimately, examining brain function in individuals with a history of MDD may contribute to the identification of endophenotypes predictive of disease onset prior to the manifestation of depressive symptoms.

Euthymic individuals with a history of MDD show a range of altered patterns of brain functioning (Farb et al., 2011; Hooley et al., 2009, 2005; Kerestes et al., 2012; Liotti et al., 2002; McCabe et al., 2009; Norbury et al., 2010; Thomas et al., 2011). Of relevance to the current study, a growing literature has demonstrated that rMDD is characterized by lower frontostriatal reactivity to negative emotional stimuli relative to never depressed controls. Specifically, rMDD showed reduced dorsolateral prefrontal cortex (DLPFC) activation to maternal criticism (Hooley et al., 2009, 2005), decreased hippocampus, insula, putamen, fusiform gyrus, and inferior frontal gyrus activation to negative emotional faces (Thomas et al., 2011), decreased medial prefrontal cortex activation to sad film clips (Farb et al., 2011), and decreased pregenual anterior cingulate cortex activation to sad autobiographical memories (Liotti et al., 2002). However, a few studies have also demonstrated increased brain activity in rMDD in response to negative emotional stimuli. For example, rMDD showed greater left DLPFC activity to negative emotional distractors during a working 
memory task (Kerestes et al., 2012), greater bilateral DLPFC activity to fearful faces (Norbury et al., 2010), higher calcarine cortex activity to sad film clips (Farb et al., 2011), and greater activation of the bilateral caudate to the sight of moldy strawberries (McCabe et al., 2009). The discrepant findings of prior studies may be attributed to differences in subthreshold depressive symptoms and ongoing psychotropic medication use between rMDD control groups. To address these limitations, the current study will focus on euthymic individuals with a history of depression who are medication-free.

Our research group recently examined neural responses to monetary gains in rMDD (Dichter et al., 2012). We found increased activation during reward gain anticipation and decreased activation during reward gain outcomes in prefrontal cortex and striatal areas in rMDD. The purpose of the current study was to extend this line of research to examine loss anticipation and outcomes in rMDD. Based on prior research showing decreased activity to loss in patients with current MDD and decreased activity to negative emotional stimuli in individuals with rMDD (Hooley et al., 2009, 2005; Knutson et al., 2008; Thomas et al., 2011), we hypothesized that the rMDD group would be characterized by reduced frontostriatal activation during both loss anticipation and outcomes. Secondary analyses explored the association between neural responses to losses and self-reported rumination in the rMDD group.

\section{Method}

\section{Participants and Procedures}

Nineteen euthymic adults with remitted major depressive disorder (rMDD) (4 male; 13 Caucasian; $23.6 \pm 4.1$ years old; 17 right-handed), based on DSM-IV diagnosis, were recruited from the Cognitive Behavioral Research and Treatment Program at Duke University Medical Center. Nineteen affectively healthy adult controls (7 male; 15 Caucasian; $27.9 \pm 6.3$ years old; all right-handed), free of lifetime MDD, were recruited from lists of potential participants maintained by the Duke-UNC Brain Imaging and Analysis Center (BIAC). After providing a complete description of the study to the participants, written informed consent was obtained.

The Structured Clinical Interview for DSM-IV-TR Axis I Disorders (SCID) (First et al., 2002) was used to confirm inclusion and exclusion criteria for both groups. Additional exclusion criteria for both groups included age $<19$ or $>55$ years, current Axis I psychopathology, psychiatric medication use within the past month, estimated verbal IQ scores $<80$, BDI $>8$, or MRI contraindications.

Estimated IQ was assessed using the North American Adult Reading Test (NAART). Depressive symptoms were assessed using the Beck Depression Inventory-II (BDI-II) (Spasojević and Alloy, 2001) and rumination was assessed using the Ruminative Responses Scale (RRS) (Nolen-Hoeksema and Morrow, 1991). The RRS is a 22-item self-report questionnaire used to assess trait rumination. Participants indicated their general tendency (i.e., never, sometimes, often, or always) to engage in ruminative behaviors (e.g., "Go someplace alone and think about your feelings") and cognitions (e.g., "Think "What am I doing to deserve this?"') in response to negative emotions.

Clinical characteristics are reported in Table 1. Groups did not differ in age, estimated verbal IQ, or gender distribution, $p$ 's > .07. By design, groups did not differ in BDI-II scores, $p>$.30; however, participants with rMDD reported significantly more rumination than the control participants $p<.05$. 
fMRI task

The fMRI task was a modified MID task (Knutson et al., 2000). Participants practiced the task outside the scanner prior to the scan session and completed four functional imaging runs. On two runs, money could be lost or not lost, but money could not be won; on the other two runs, money could be won or not won, but money could not be lost. Consistent with our aim of characterizing neural responses to loss in rMDD, only results from runs with potential monetary losses are presented here. Results from runs with potential monetary gain have been reported previously (Dichter et al., 2012). Each run began with a 10-s instructional screen indicating whether the forthcoming run would be a "win" or a "loss" run. Run types (i.e., win or loss runs) were presented in alternating order, and the run type presented first was counterbalanced across participants.

Task conditions and trial timings are summarized in Figure 1. Each trial consisted of: 1) a $2000 \mathrm{~ms}$ cue that indicated whether a slow response to the forthcoming target bulls-eye could result in a "loss" (a triangle) or no loss (a circle); 2) a delay period during which a crosshair was presented for 2000-2500 ms; 3) a target bulls-eye that required a speeded button press presented for up to $500 \mathrm{~ms}$; 4) $3000 \mathrm{~ms}$ of feedback that indicated whether that trial was a "loss" or not; and 5) a variable length ITI crosshair presented such that the total duration of each trial was $12 \mathrm{~s}$. Trial types (i.e., potential loss or not potential loss) were aperiodic and pseudorandomly ordered (Knutson et al., 2000).

Participants could lose $\$ 1$ per trial, and feedback was a text display of the amount of money lost (e.g., "-\$1"). Coincident with this feedback, a cumulative count of the number of dollars lost within the run was presented. Participants were instructed to respond to all target bullseyes as quickly as possible, and outcomes were contingent on reaction times. The task was adaptive such that participants were successful on approximately two-thirds of trials, regardless of individual differences in reaction times. Each 8-minute run contained 40 trials: 20 were potential loss trials, 20 were non-loss trials. Prior to entering the scanner, participants were shown the money they could lose based on scanner task performance. Stimuli were presented using E-Prime presentation software v.1.1 (Psychology Software Tools Inc. Pittsburgh, PA) and displayed in the scanner though magnet-compatible goggles (Resonance technology, Inc., Northridge, CA). Reaction times were recorded on each trial of the MID task.

\section{Imaging methods}

Scanning was performed on a General Electric (Waukesha, Wisconsin, USA) MR750 3.0 Tesla scanner with a quadrature birdcage radio frequency head coil. This scanner is equipped with high-power heavy-duty-cycle-50-mT/m gradients at a $200 \mathrm{~T} / \mathrm{m} / \mathrm{s}$ slew rate and a 32-channel head coil for parallel imaging. A high-resolution T1-weighted image with 166 slices was acquired using a 3D FSPGR pulse sequence (TR=7.484 ms; TE=2.984 ms; FOV $=256 \mathrm{~mm}$; image matrix $=256 \times 256$; voxel size $=1 \mathrm{~mm}^{3}$ ) and used for coregistration with the functional data. This structural image was aligned in a near axial plane defined by the anterior and posterior commissures. Whole brain functional images were acquired using a spiral pulse sequence with SENSE reconstruction sensitive to blood oxygenation level dependent contrast (TR, $2000 \mathrm{~ms}$; TE, $30 \mathrm{~ms}$; FOV, $256 \mathrm{~mm}$; image matrix, $64 \times 64$; $\mathrm{a}=60^{\circ}$; voxel size $=4 \mathrm{~mm}^{3} ; 32$ axial slices). Functional images were aligned similarly to the T1weighted structural image. A semi-automated high-order shimming program ensured global field homogeneity.

\section{Imaging data analysis}

Functional data were preprocessed using FSL version 4.1.8 (Oxford Centre for Functional Magnetic Resonance Imaging of the Brain (FMRIB), Oxford University, U.K.). Timing files 
were converted to FSL-compatible format and NIFTI image data files were generated. The following preprocessing steps were implemented using the FSL FEAT preprocessing utility: (i) brain extraction for non-brain removal (Smith et al., 2004), (ii) motion correction using MCFLIRT (Smith, 2002), (iii) spatial smoothing using a Gaussian kernel of FWHM 5 mm, (iv) mean-based intensity normalization of all volumes by the same factor, and (v) high-pass filtering (Jenkinson et al., 2002). Functional images were co-registered to structural images in native space for each participant, and structural images were normalized into a standard stereotaxic space (Montreal Neurological Institute) for inter-participant comparison. The same transformation matrices used for structural-to-standard transformations were then used for functional-to-standard space transformations of co-registered functional images. All registrations were carried out using an intermodal registration tool (Jenkinson et al., 2002; Smith et al., 2004). Voxel-wise temporal autocorrelation was estimated and corrected using FMRIB's Improved Linear Model (FILM) (Jenkinson and Smith, 2001).

Onset times of events were used to model a signal response containing a regressor for each response type, which was convolved with a double- $y$ function to model the hemodynamic response. Group-wise activation images were calculated by a mixed effects higher-level analysis using Bayesian estimation techniques with FMRIB Local Analysis of Mixed Effects (FLAME) (Smith et al., 2004; Woolrich et al., 2001). Key anatomical regions within the reward system (frontal lobes, amygdala, nucleus accumbens, insula, thalamus, caudate nucleus, anterior cingulate gyrus, and putamen) that have previously been found to be functionally impaired in MDD (Davidson et al., 2002a; Knutson et al., 2008) were defined a priori for small volume correction. These regions were generated in FSL using the HarvardOxford cortical and subcortical structural probabilistic atlases. All masks were thresholded at $25 \%$, binarized, and then combined into a single mask using fslmaths.

The anticipation and outcome phases of the task were analyzed separately. For both phases, the primary method of analysis was to identify clusters that showed significant interactions of Group (control and rMDD) and trial type (potential loss and non-potential loss during the anticipatory phase, and losses and non-losses during the outcome phase). For all analyses, voxels were considered significant if they passed a statistical threshold of $p<.005$, uncorrected, and were part of a 39-voxel cluster of contiguous significant voxels, resulting in a cluster-corrected $p<.05$. This cluster size was determined by performing 1000 Monte Carlo simulations using 3dClustSim (Ward, 2000).

\section{Results}

\section{Head Motion}

There were no significant differences between groups in mean displacement across three dimensions (X, Y, and $\mathrm{Z}$ ) on any of the runs (all $p$ 's $>.16$ ).

\section{In-Scanner Reaction Times}

As shown in Table 1, participants with rMDD showed slower reaction times than controls when anticipating potential loss, $p<.05$. Groups did not differ in reaction times on non-loss trials, $p=.50$.

\section{Brain Activation}

Clusters with significant activation differences between rMDD and control participants during both loss anticipation and outcome are shown in Table 2. During anticipation of loss, relative to anticipation of non-loss, rMDD participants showed relatively less activation of the left superior frontal gyrus (see Figure 2). During loss outcomes, relative to non-loss outcomes, rMDD participants showed relatively less activation of the left inferior frontal 
gyrus and left superior frontal gyrus (see Figure 2). There were no clusters with significantly greater activation in the rMDD group than the control group.

\section{Correlations with Task Behavior and Rumination}

Correlations between the magnitude of brain activation in clusters showing significant differences between groups (i.e., the superior frontal gyrus during loss anticipation depicted in Figure 2 and the inferior and superior frontal gyri during loss outcomes depicted in Figure 2), reaction times, and RRS scores were examined in the rMDD group (see Table 2). Because six correlations were examined (i.e., between activations in three clusters with RT and RRS values), these correlations were evaluated for significance at a Bonferronicorrected statistical threshold of $=.0083$. Activation in the left superior frontal gyrus during loss outcomes (relative to non-loss outcomes) were negatively correlated with RRS scores in the rMDD group, $r=-.68 . p<.0015$ (see Figure 3).

\section{Discussion}

The current examination of loss processing in rMDD extends our previous research on reward gain processing in rMDD (Dichter et al., 2012) and complements prior research on the neural characteristics of depression risk (Farb et al., 2011; Hooley et al., 2009, 2005; Thomas et al., 2011). Our prior research on reward gains showed abnormal neural responses during both reward gain anticipation and outcomes in rMDD (Dichter et al., 2012), and as such, we expected to see group differences during both loss anticipation and outcomes. Consistent with our hypothesis, rMDD showed significantly reduced brain activation compared to controls during both loss anticipation and outcomes. The reduced left inferior and superior frontal gyri reactivity to loss outcomes seen in rMDD in the current study was consistent with previous studies demonstrating reduced prefrontal cortex reactivity to fear, sadness, and criticism in rMDD (Farb et al., 2011; Hooley et al., 2009, 2005; Thomas et al., 2011). Loss anticipation and outcomes have been shown to elicit negative affective arousal (Knutson and Greer, 2008). Taken together, these results suggest that MDD risk is characterized by reduced prefrontal cortex reactivity to negative affective states.

The left superior frontal gyrus is involved in both working memory and self-awareness (Boisgueheneuc et al., 2006; Goldberg et al., 2006). Recent research in healthy individuals suggests that the superior frontal gyrus becomes inactive during cognitively demanding perceptual tasks during which reflective self-awareness is diminished (Goldberg et al., 2006). One interpretation of the reduced superior frontal gyrus activity in rMDD is therefore that the reward trials were more cognitively demanding for rMDD than controls, which resulted in a diminished cognitive capacity to engage in self-awareness. This interpretation is supported by the slower reaction times to targets following loss cues but not non-loss (neutral) cues in rMDD, which suggests that increased cognitive demand was specific to the loss trials rather than the task as a whole. Thus, reduced superior frontal gyrus activity during loss trials may reflect increased cognitive load associated with rumination. Indeed, lower superior frontal gyrus activity during loss outcomes was associated with greater trait rumination in rMDD.

We also found that individuals with rMDD demonstrated significantly lower activation in the inferior frontal gyrus (BA 45), an area associated with semantic processing (Liakakis et al., 2011) and negative affect (Kret et al., 2011). In a prior fMRI study, negative emotional load during a cognitive task that involved making judgments about images was associated with attenuated activation of the ventral part of the inferior frontal gyrus (Northoff et al., 2004). Thus, inferior frontal gyrus activity may be attenuated in rMDD as a result of an increased sensitivity to the emotional load associated with loss. Moreover, a prior study of personality and neural responsivity to negative emotional faces demonstrated 
hyporesponsivity in BA 45 among individuals with higher levels of negative affect (Kret et al., 2011). Thus, reduced inferior frontal gyrus responsivity to loss in the current study may constitute a neural biomarker of trait negative affectivity and depression vulnerability.

Comparing the present findings with those reported in Dichter et al (2012), it is noteworthy that neural responses to loss may be distinguished from neural responses to gain in rMDD. Whereas across studies the rMDD group was characterized by reduced activation in frontal regions during both loss anticipation and outcomes, responses during monetary gain were characterized by frontal cortex hyperactivation during reward anticipation and hypoactivation during reward outcomes (Dichter et al., 2012). Additionally, none of the brain areas associated with group differences between rMDD and controls in response to gain were associated with rumination (Dichter et al., 2012). One interpretation is therefore that the loss condition was more sensitive to ruminative response styles associated with rMDD, thus explaining the hypoactivation in frontal areas associated with attention and cognitive processing. Nonetheless, these results make clear that examining the full chronometry of the reward response, both anticipation and experience of gains and losses, is essential to uncover subtle differences between rMDD and controls.

Additionally, the left lateralization of prefrontal cortex hypoactivity in rMDD seen in the current study is consistent with prior EEG research linking left frontal hypoactivity, behavioral approach deficits, and MDD (Davidson, 1998; Davidson et al., 2002b; Tomarken et al., 2004). Davidson, Tomarken, and colleagues have posited that left prefrontal cortex hypoactivity is a depressogenic vulnerability that, when combined with negative life events, increases the probability of developing MDD (Davidson, 1998). One way to study firstonset MDD risk is to examine prospectively a group of adolescents who have never experienced depression but who have family members with depression. Consistent with Davidson's model, euthymic adolescents, whose mothers had a history of depression, showed left frontal hypoactivity during resting-state EEG (Tomarken et al., 2004). Taken together, the current fMRI and previous EEG findings suggest that left prefrontal cortex is underactive during loss processing and hypoactive at rest. Given that left lateralized prefrontal cortex activity is seen as a marker of effective coping and resilience to affective illness (Davidson, 2004), the left hypofrontality seen in the current study in response to both loss anticipation and outcomes may therefore reflect a trait vulnerability to depression. Further research is needed to explore the peripheral neuroendocrine and immune function associated with left frontal hyporeactivity to loss that would be predicted by Davidson's conceptualization of resilience (Davidson, 2004) and to determine whether individual differences in left frontal hypoactivity in rMDD can be used to explain variance in relapse rates.

Results of the current study also demonstrate a significant association between neural response to loss and rumination in the rMDD group. Rumination, defined as the tendency to perseverate on negative emotions, characterizes chronic, severe depression (NolenHoeksema, 2000) and increases the risk for depression following loss (Nolen-Hoeksema et al., 1994). In prior studies, RRS scores have been found to be positively correlated with reactivity of the inferior frontal gyrus to fearful faces (Thomas et al., 2011) and medial prefrontal cortex to sad film clips (Farb et al., 2011). However, in the current study, we observed a negative correlation between RRS scores and superior frontal gyrus reactivity to loss outcomes. Differences inherent in the study designs, such as the elicitation of brain activation by varying methods (e.g., fearful faces, sad films, or monetary loss) and clinical characteristics of the rMDD group (e.g., the inclusion of those with significantly elevated depressive symptoms and currently using psychotropic medications), may explain the differences in findings. However, the results of these studies generally suggest that depression risk, reduced frontal reactivity to negative emotions, and rumination are 
interrelated. Although our analyses cannot provide information about the direction of the effects, rumination mediated the association between prefrontal cortex activity and depression relapse in rMDD in a previous study (Farb et al., 2011). Thus, follow-up studies will be necessary to determine whether the depressogenic effects of reduced frontal reactivity to loss are mediated by rumination.

Limitations of this study include a small sample size, the inclusion of two participants with rMDD who were receiving psychotherapy at the time of participation, and a history of psychotropic medication in five participants with rMDD. Although the neural correlates of MDD risk were examined without the confounding effects of current depressive symptoms or medication use, further research is needed to evaluate the utility of this functional neural endophenotype as a prospective marker of MDD risk.

In summary, our findings suggest that currently euthymic individuals with a history of MDD are characterized by reduced superior frontal gyrus activation during loss anticipation and reduced activity of both the inferior and superior frontal gyri during loss outcomes. In the context of a growing body of literature demonstrating reduced prefrontal cortex reactivity to negative emotions in rMDD, these results highlight the importance of examining neural responses to loss, in addition to gains, when studying reward network dysfunction in MDD. More broadly, these findings suggest that aberrant prefrontal cortex response to loss may represent a trait endophenotype for MDD, although future studies in high-risk groups, particularly those without a history of MDD, will be critical to establish this as a prospective marker of MDD vulnerability.

\section{Acknowledgments}

This research was supported by grants from the NARSAD Young Investigator Program. Investigator effort was supported by NIMH T32 MH093315 (C. Schiller, trainee), NIMH K23 MH087754 (M. Smoski), and NIMH K23 MH081285 (G. Dichter), and R21 MH094781 (M. Smoski, G. Dichter, \& J. Minkel). Assistance for this study was provided by the Neuroimaging Core of the Carolina Institute for Developmental Disabilities (P30 HD03110).

The authors thank Dr. Brian Knutson for kindly sharing the MID task, Josh Bizzell, Chris Petty, Allison Rittenberg, John Anthony Richey, and Todd Harshbarger for assistance with image analysis, MRI technologists Susan Music, Natalie Goutkin, and Luke Poole for assistance with data acquisition, and BIAC Director Allen Song for assistance with various aspects of this project.

\section{References}

Boisgueheneuc, F du; Levy, R.; Volle, E.; Seassau, M.; Duffau, H.; Kinkingnehun, S.; Samson, Y.; Zhang, S.; Dubois, B. Functions of the left superior frontal gyrus in humans: a lesion study. Brain. 2006; 129:3315-3328. [PubMed: 16984899]

Choi JS, Shin YC, Jung WH, Jang JH, Kang DH, Choi CH, Choi SW, Lee JY, Hwang JY, Kwon JS. Altered Brain Activity during Reward Anticipation in Pathological Gambling and ObsessiveCompulsive Disorder. Plos One. 2012; 7:e45938. [PubMed: 23029329]

Davidson RJ. Anterior electrophysiological asymmetries, emotion, and depression: Conceptual and methodological conundrums. Psychophysiology. 1998; 35:607-614. [PubMed: 9715104]

Davidson RJ. What does the prefrontal cortex "do" in affect: perspectives on frontal EEG asymmetry research. Biol Psychol. 2004; 67:219-234. [PubMed: 15130532]

Davidson RJ, Lewis DA, Alloy LB, Amaral DG, Bush G, Cohen JD, Drevets WC, Farah MJ, Kagan J, McClelland JL, Nolen-Hoeksema S, Peterson BS. Neural and behavioral substrates of mood and mood regulation. Biol Psychiatry. 2002a; 52:478-502. [PubMed: 12361665]

Davidson RJ, Pizzagalli D, Nitschke JB, Putnam K. Depression: Perspectives from Affective Neuroscience. Annu Rev Psychol. 2002b; 53:545-574. [PubMed: 11752496] 
Dichter GS, Kozink RV, McClernon FJ, Smoski MJ. Remitted major depression is characterized by reward network hyperactivation during reward anticipation and hypoactivation during reward outcomes. J Affect Disord. 2012; 136:1126-1134. [PubMed: 22036801]

Farb NA, Anderson AK, Bloch RT, Segal ZV. Mood-linked responses in medial prefrontal cortex predict relapse in patients with recurrent unipolar depression. Biol Psychiatry. 2011; 70:366-72. [PubMed: 21531382]

First, MB.; Spitzer, RL.; Gibbon, M.; Williams, JBW. Biometrics Research. New York State Psychiatric Institute; New York: 2002. Structured Clinical Interview for DSM-IV-TR Axis I Disorders, Research Version, Non-patient Edition. (SCID-I/NP).

Forbes EE, Hariri AR, Martin SL, Silk JS, Moyles DL, Fisher PM, Brown SM, Ryan ND, Birmaher B, Axelson DA, Dahl RE. Altered Striatal Activation Predicting Real-World Positive Affect in Adolescent Major Depressive Disorder. Am J Psychiatry. 2009; 166:64-73. [PubMed: 19047324]

Goldberg II, Harel M, Malach R. When the Brain Loses Its Self: Prefrontal Inactivation during Sensorimotor Processing. Neuron. 2006; 50:329-339. [PubMed: 16630842]

Hooley JM, Gruber SA, Parker HA, Guillaumot J, Rogowska J, Yurgelun-Todd DA. Cortico-limbic response to personally challenging emotional stimuli after complete recovery from depression. Psychiatry Res Neuroimaging. 2009; 172:83-91.

Hooley JM, Gruber SA, Scott LA, Hiller JB, Yurgelun-Todd DA. Activation in dorsolateral prefrontal cortex in response to maternal criticism and praise in recovered depressed and healthy control participants. Biol Psychiatry. 2005; 57:809-12. [PubMed: 15820239]

Jenkinson M, Bannister P, Brady M, Smith S. Improved Optimization for the Robust and Accurate Linear Registration and Motion Correction of Brain Images. NeuroImage. 2002; 17:825-841. [PubMed: 12377157]

Jenkinson M, Smith S. A global optimisation method for robust affine registration of brain images. Med Image Anal. 2001; 5:143-156. [PubMed: 11516708]

Keedwell PA, Andrew C, Williams SCR, Brammer MJ, Phillips ML. The neural correlates of anhedonia in major depressive disorder. Biol Psychiatry. 2005; 58:843-853. [PubMed: 16043128]

Kendler KS, Hettema JM. Life event dimensions of loss, humiliation, entrapment, and danger in the prediction of onsets of major depression and generalized anxiety. Arch Gen Psychiatry. 2003; 60:789-796. [PubMed: 12912762]

Kerestes R, Ladouceur CD, Meda S, Nathan PJ, Blumberg HP, Maloney K, Ruf B, Saricicek A, Pearlson GD, Bhagwagar Z, Phillips ML. Abnormal prefrontal activity subserving attentional control of emotion in remitted depressed patients during a working memory task with emotional distracters. Psychol Med. 2012; 42:29-40. [PubMed: 21733287]

Knutson B, Bhanji JP, Cooney RE, Atlas LY, Gotlib IH. Neural responses to monetary incentives in major depression. Biol Psychiatry. 2008; 63:686-92. [PubMed: 17916330]

Knutson B, Greer SM. Anticipatory affect: neural correlates and consequences for choice. Philos Trans R Soc B Biol Sci. 2008; 363:3771-3786.

Knutson B, Westdorp A, Kaiser E, Hommer D. FMRI visualization of brain activity during a monetary incentive delay task. Neuroimage. 2000; 12:20-27. [PubMed: 10875899]

Kret ME, Denollet J, Grèzes J, de Gelder B. The role of negative affectivity and social inhibition in perceiving social threat: An fMRI study. Neuropsychologia. 2011; 49:1187-1193. [PubMed: 21315749]

Liakakis G, Nickel J, Seitz RJ. Diversity of the inferior frontal gyrus-A meta-analysis of neuroimaging studies. Behav Brain Res. 2011; 225:341-347. [PubMed: 21729721]

Liotti M, Mayberg HS, McGinnis S, Brannan SL, Jerabek P. Unmasking Disease-Specific Cerebral Blood Flow Abnormalities: Mood Challenge in Patients With Remitted Unipolar Depression. Am J Psychiatry. 2002; 159:1830-1840. [PubMed: 12411216]

McCabe C, Cowen PJ, Harmer CJ. Neural representation of reward in recovered depressed patients. Psychopharmacology (Berl). 2009; 205:667-677. [PubMed: 19529923]

Mitterschiffthaler MT, Kumari V, Malhi GS, Brown RG, Giampietro VP, Brammer MJ, Suckling J, Poon L, Simmons A, Andrew C, Sharma T. Neural response to pleasant stimuli in anhedonia: an fMRI study. Neuroreport. 2003; 14:177-182. [PubMed: 12598724] 
Nolen-Hoeksema S. The role of rumination in depressive disorders and mixed anxiety/depressive symptoms. J Abnorm Psychol. 2000; 109:504-511. [PubMed: 11016119]

Nolen-Hoeksema S, Morrow J. A prospective study of depression and posttraumatic stress symptoms after a natural disaster: the 1989 Loma Prieta Earthquake. J Pers Soc Psychol. 1991; 61:115-121. [PubMed: 1890582]

Nolen-Hoeksema S, Parker LE, Larson J. Ruminative coping with depressed mood following loss. J Pers Soc Psychol. 1994; 67:92-104. [PubMed: 8046585]

Norbury R, Selvaraj S, Taylor MJ, Harmer C, Cowen PJ. Increased neural response to fear in patients recovered from depression: a 3T functional magnetic resonance imaging study. Psychol Med. 2010; 40:425-432. [PubMed: 19627640]

Northoff G, Heinzel A, Bermpohl F, Niese R, Pfennig A, Pascual-Leone A, Schlaug G. Reciprocal modulation and attenuation in the prefrontal cortex: An fMRI study on emotional-cognitive interaction. Hum Brain Mapp. 2004; 21:202-212. [PubMed: 14755839]

Schaefer HS, Putnam KM, Benca RM, Davidson RJ. Event-Related Functional Magnetic Resonance Imaging Measures of Neural Activity to Positive Social Stimuli in Pre- and Post-Treatment Depression. Biol Psychiatry. 2006; 60:974-986. [PubMed: 16780808]

Smith SM. Fast robust automated brain extraction. Hum Brain Mapp. 2002; 17:143-155. [PubMed: 12391568]

Smith SM, Jenkinson M, Woolrich MW, Beckmann CF, Behrens TEJ, Johansen-Berg H, Bannister PR, De Luca M, Drobnjak I, Flitney DE, Niazy RK, Saunders J, Vickers J, Zhang Y, De Stefano N, Brady JM, Matthews PM. Advances in functional and structural MR image analysis and implementation as FSL. NeuroImage. 2004; 23:S208-S219. [PubMed: 15501092]

Smoski MJ, Rittenberg A, Dichter GS. Major depressive disorder is characterized by greater reward network activation to monetary than pleasant image rewards. Psychiatry Res Neuroimaging. 2011; 194:263-270.

Spasojević J, Alloy LB. Rumination as a common mechanism relating depressive risk factors to depression. Emotion. 2001; 1:25-37. [PubMed: 12894809]

Thomas EJ, Elliott R, McKie S, Arnone D, Downey D, Juhasz G, Deakin JFW, Anderson IM. Interaction between a history of depression and rumination on neural response to emotional faces. Psychol Med. 2011; 41:1845-1855. [PubMed: 21306660]

Tomarken AJ, Dichter GS, Garber J, Simien C. Resting frontal brain activity: linkages to maternal depression and socio-economic status among adolescents. Biol Psychol. 2004; 67:77-102. [PubMed: 15130526]

Ward, BD. Simultaneous inference for fMRI data. Biophysics Research Institute, Medical College of Wisconsin; Milwaukee: 2000.

Woolrich MW, Ripley BD, Brady M, Smith SM. Temporal Autocorrelation in Univariate Linear Modeling of FMRI Data. NeuroImage. 2001; 14:1370-1386. [PubMed: 11707093] 


\section{Loss Trials $\quad \underline{\text { Non-Loss Trials }}$}

$\underline{\text { Cue }}$
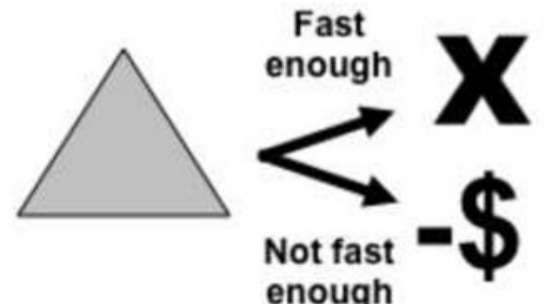

$\underline{\text { Cue }}$

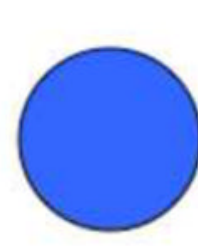

Fast enough

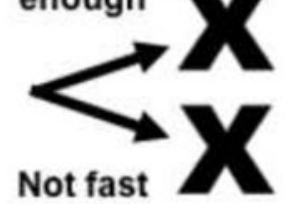

enough

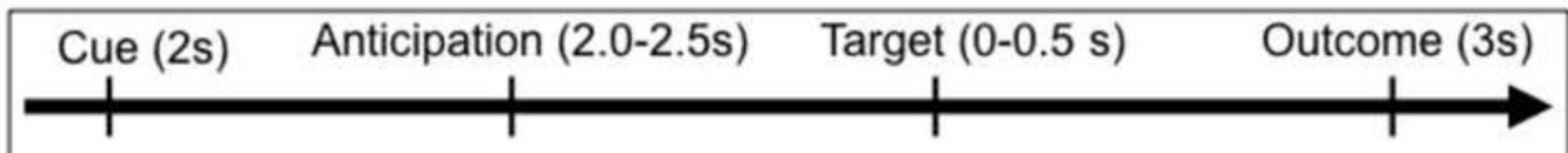

Figure 1.

The MID task. Each trial consisted of a cue (i.e., a triangle indicated an incentive trial, a circle indicated a non-incentive trial), an anticipatory delay, a target, and outcome feedback. 


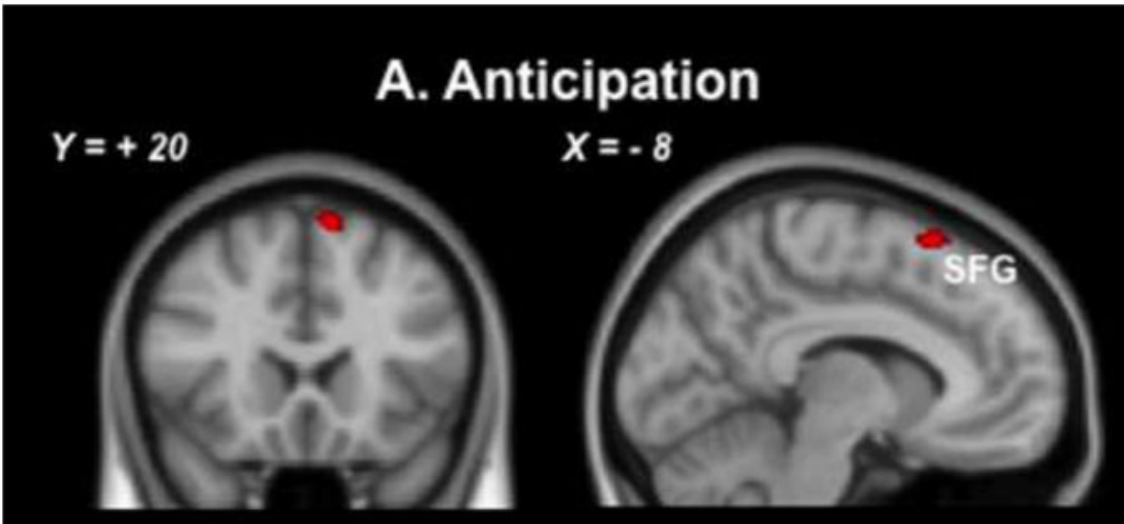

\section{B. Outcome}

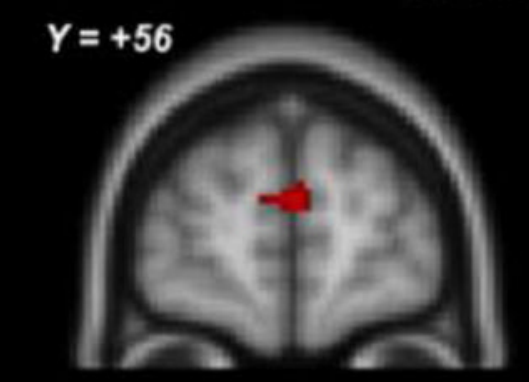

$$
x=-4
$$
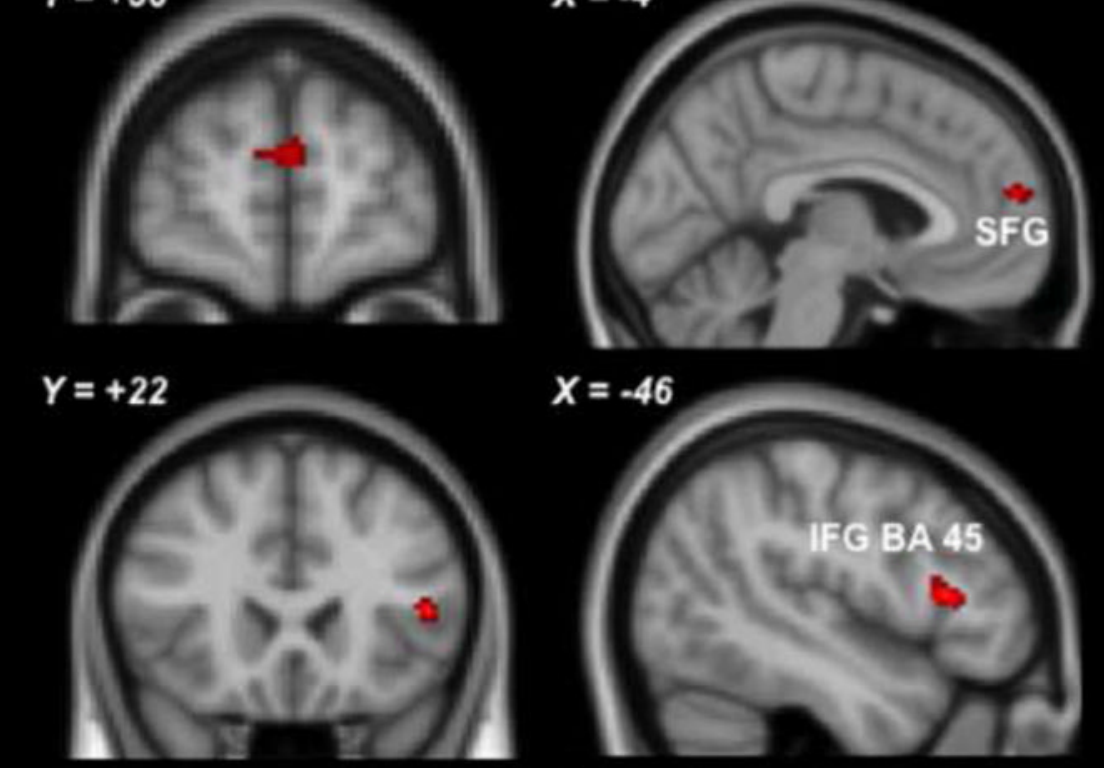

$X=-46$

\section{Control > Remitted 2.58

Figure 2.

Coronal and sagittal images depicting significantly less activation prefrontal cortex activation in rMDD versus controls during (A) loss anticipation relative to non-loss anticipation and (B) loss outcomes relative to non-loss outcomes. Statistical images were thresholded using a cluster corrected significance threshold of $p<.05$. Coordinates are in Montreal Neurological Institute space. $\mathrm{SFG}=$ superior frontal gyrus; IFG=inferior frontal gyrus. 


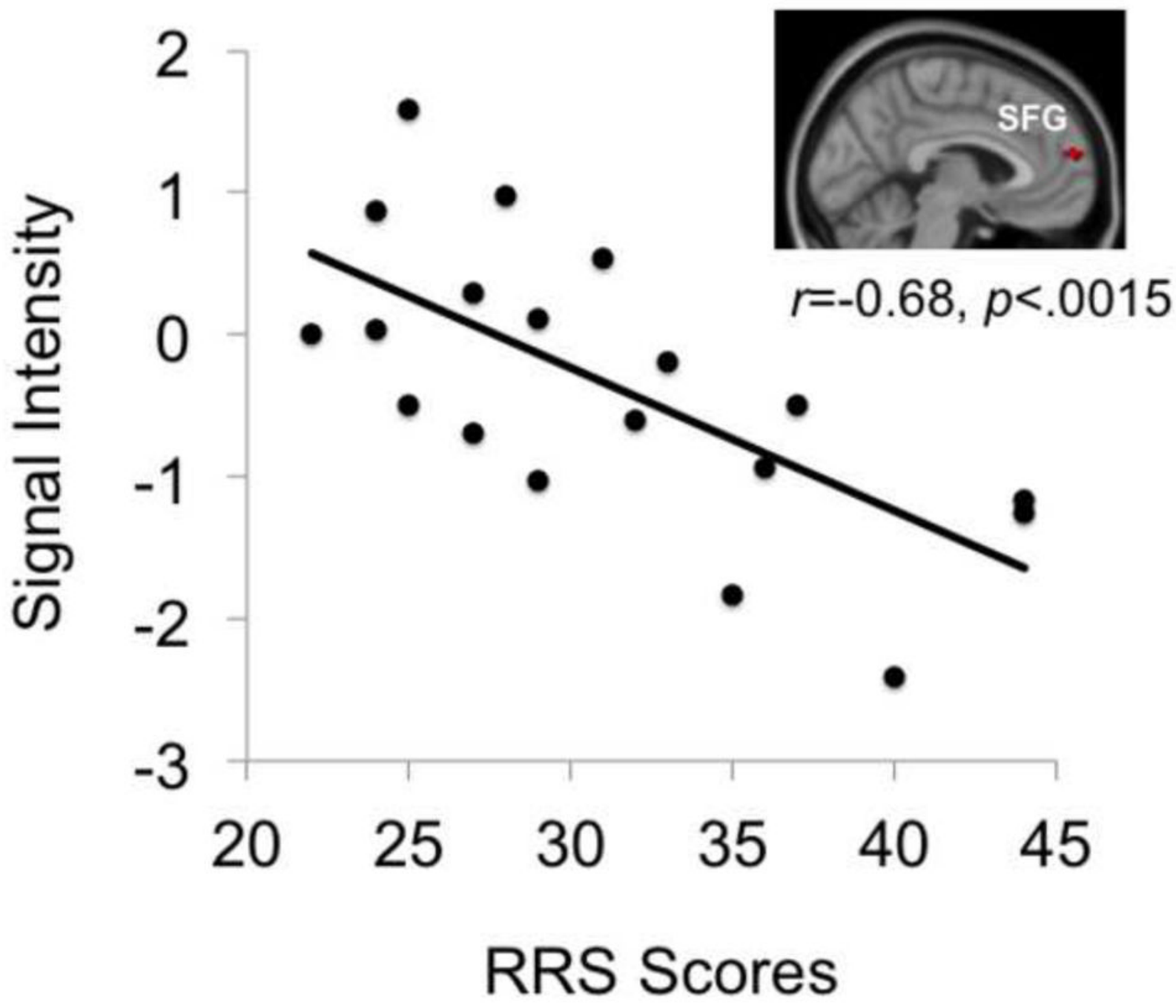

Figure 3.

Correlation between activation of the left superior frontal gyrus during loss outcomes and RRS scores in the rMDD group. SFG=superior frontal gyrus. 


\section{Table 1}

Demographic and clinical characteristics of control and rMDD participants.

\begin{tabular}{|c|c|c|c|}
\hline & $\begin{array}{c}\operatorname{rMDD}(n=19 ; 4 \text { ठ }) \\
M(S D)\end{array}$ & $\begin{array}{c}\text { Controls }(n=19 ; 7 \text { f) } \\
M(S D)\end{array}$ & $t(p)$ \\
\hline Age & $23.6(4.1)$ & $27.9(6.3)$ & $1.9(0.072)$ \\
\hline RRS & $1.4(0.3)$ & $1.3(0.2)$ & $2.1(0.045)$ \\
\hline NAART: Verbal IQ & $110.4(5.0)$ & $110.7(3.3)$ & $0.2(0.81)$ \\
\hline BDI-II & $2.6(4.9)$ & $1.4(2.3)$ & $1.0(0.32)$ \\
\hline Number with a prior hospitalization for MDD & 3.0 & 0.0 & \\
\hline Past MDD episodes & $1.6(0.9)$ & 0.0 & \\
\hline Duration in months of most recent MDD episode & $6.8(5.2)$ & 0.0 & \\
\hline Number of months since most recent MDD episode & $40.8(44.9)$ & 0.0 & \\
\hline Reaction time on loss trials (ms) & $201.1(19.3)$ & $186.4(19.0)$ & $2.4(0.02)$ \\
\hline Reaction time on non-loss trials (ms) & $224.1(32.3)$ & $217.2(30.5)$ & $0.7(0.5)$ \\
\hline
\end{tabular}

Note. rMDD = remitted major depressive disorder; RSS = Ruminative Responses Scale; NAART = North American Adult Reading Test; BDI-II = Beck Depression Inventory-II; MDD = Major Depressive Disorder 


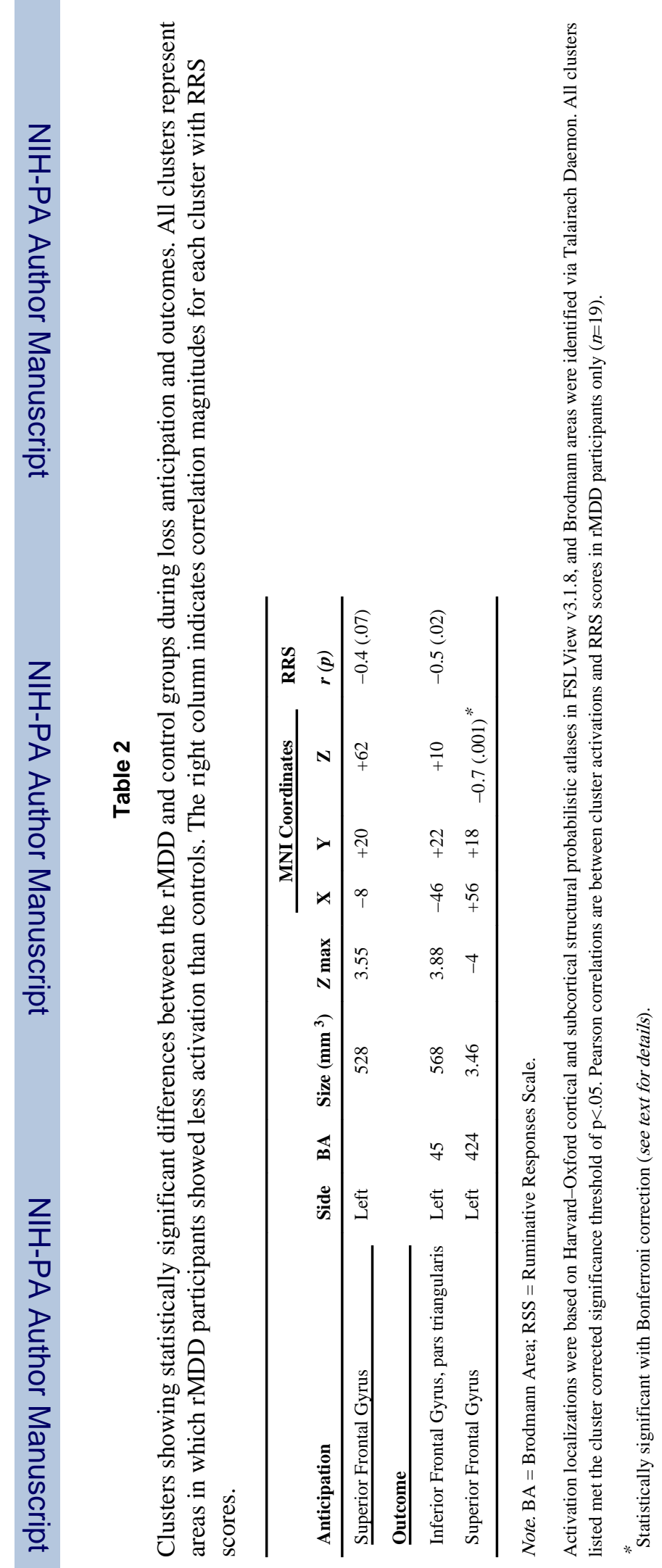

